\title{
Kikuchi-Fujimoto disease presenting as brainstem encephalitis with secondary blepharospasm
}

\author{
Dushyanth Babu Jasti, S. V. Naveen Prasad, Thota Naveen, Bhuma Vengamma \\ Department of Neurology, SVIMS, Tirupati, Andhra Pradesh, India
}

\begin{abstract}
Central nervous system involvement in Kikuchi-Fujimoto disease is a very rare clinical manifestation. We report a 15-year-old girl who presented to us with fever, drowsiness, neck swellings, and involuntary closure of both eyelids of 2 days duration. Magnetic resonance imaging brain showed T2-weighted and fluid-attenuated inversion recovery hyperintensities in dorsal midbrain and pons. Cervical lymph node fine-needle aspiration cytology was suggestive of Kikuchi-Fujimoto disease. Blepharospasm secondary to infectious etiology is rare. Positron emission computed tomography brain showed increased focal uptake in anterior cingulate gyrus which can be the site of origin of blepharospasm. The patient was managed with steroids and trihexyphenidyl with significant recovery. Kikuchi-Fujimoto disease is a rare disease which has to be considered as one of the differential diagnosis in a case of acute encephalopathy with cervical lymphadenopathy.
\end{abstract}

Key words: Blepharospasm, encephalitis, Kikuchi-Fujimoto disease, neurological manifestations

\section{Introduction}

Kikuchi-Fujimoto disease is a benign lymphadenitis which usually resolves spontaneously. It is a rare disease seen in Asian adults. It was first reported in Japan in 1972. ${ }^{[1]}$ It is also known as histiocytic necrotizing lymphadenitis. ${ }^{[2]} \mathrm{It}$ is of clinical importance because it is often misdiagnosed as tuberculosis or lymphoma. Common clinical features include cervical lymph node enlargement and fever which can mimic tuberculosis. Kikuchi-Fujimoto disease constituted $2.1 \%$ of 1724 lymph node biopsy specimens in a study published from Tirupati ${ }^{[3]}$ and has been occasionally reported from India. Involvement of central nervous system (CNS) is very rare in Kikuchi-Fujimoto disease. We report this case of Kikuchi-Fujimoto disease with CNS involvement

\section{Address for correspondence:}

Dr. Dushyanth Babu Jasti, Department of Neurology, SVIMS,

Alipiri Road, Tirupati, Andhra Pradesh, India.

E-mail: dushyanthjasti@yahoo.co.in

\begin{tabular}{|l|l|}
\hline \multicolumn{2}{|c|}{ Videos avilable on: www.ruralneuropractice.com } \\
\hline \multicolumn{2}{|c|}{ Access this article online } \\
\hline Quick Response Code: & Website: \\
\hline & www.ruralneuropractice.com \\
\cline { 2 - 3 } & \\
\hline
\end{tabular}

in the form of brainstem encephalitis and secondary blepharospasm.

\section{Case Report}

A 15-year-old girl without any premorbid medical or surgical illness was brought to our hospital with complaints of fever and drowsiness of 2 days duration. She had a history of fever with multiple cervical lymph node enlargement 2 weeks ago, for which she was treated outside with oral levofloxacin $500 \mathrm{mg}$ once daily for 1-week. On examination, the patient was drowsy. She was febrile. Bilateral cervical lymphadenopathy was present involving a posterior group of cervical lymph nodes. Neurological examination showed vertical gaze palsy with bilateral horizontal gaze-evoked nystagmus. Blepharospasm was present [Videos 1 and 2]. Convergence was impaired in both eyes. Fundus examination was normal. Slit lamp examination did not show any evidence of Kayser-Fleischer ring. No focal motor deficits were noted. Bilateral knee and ankle jerks

This is an open access article distributed under the terms of the Creative Commons Attribution-NonCommercial-ShareAlike 3.0 License, which allows others to remix, tweak, and build upon the work non-commercially, as long as the author is credited and the new creations are licensed under the identical terms.

For reprints contact: reprints@medknow.com

How to cite this article: Jasti DB, Naveen Prasad SV, Naveen T, Vengamma B. Kikuchi-Fujimoto disease presenting as brainstem encephalitis with secondary blepharospasm. J Neurosci Rural Pract 2016;7:157-60. 
were sluggish with bilateral extensor plantar. Bilateral cerebellar signs and gait ataxia were present. Meningeal signs were present. Laboratory investigations showed leukopenia (total leucocyte count: 3000 cells/cu.mm). Erythrocyte sedimentation rate was $20 \mathrm{~mm} / 1^{\text {st }} \mathrm{h}$. Cerebrospinal fluid (CSF) analysis showed glucose $76 \mathrm{mg} / \mathrm{dl}$, protein $28 \mathrm{mg} / \mathrm{dl}$, and 45 white blood cells with $100 \%$ lymphocytes. Video electroencephalogram was normal. Magnetic resonance imaging of brain plain and contrast showed altered signal intensity which was hyperintense on T2-weighted [Figure 1] and fluid-attenuated inversion recovery sequences without any postcontrast enhancement in dorsal pons and midbrain [Figure 2]. CSF tuberculosis polymerase chain reaction (PCR) and Herpes simplex PCR were negative. CSF culture was sterile. CSF adenosine deaminase was 6.9 IU/L. CSF was negative for Listeria, Epstein-Barr virus, Enterovirus, and Cryptococcus. Enzyme-linked immunosorbent assay for human immunodeficiency virus, hepatitis $B$ virus, and hepatitis $C$ virus were negative. Antinuclear antibody, double-stranded DNA, and venereal disease research laboratory test were negative. Serum paraneoplastic neuronal antigen and antibody profile was negative. Mantoux test was negative. The patient was managed with intravenous ceftriaxone $2 \mathrm{~g}$ twice daily and intravenous dexamethasone $(4 \mathrm{mg}$ thrice daily) for 10 days in suspicion of brainstem encephalitis. She was managed with $0.5 \mathrm{mg}$ once daily clonazepam and $1 \mathrm{mg}$ twice daily trihexyphenidyl for 10 days for blepharospasm. Cervical lymph node fine-needle aspiration cytology was done which showed lymphocytes, sinus histiocytes, endocapillary buds, monocytoid cells, necrosis, karyorrhectic debris, and nodal reticulum suggestive of Kikuchi-Fujimoto disease [Figure 3]. Positron emission tomography (PET)

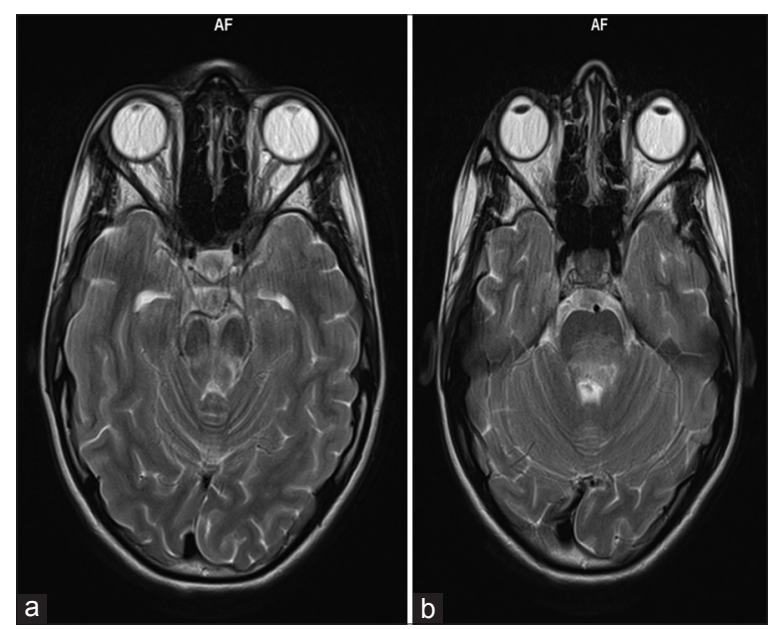

Figure 1: Magnetic resonance imaging of brain plain T2-weighted sequence showing hyperintense signal in (a) dorsal midbrain (b) dorsal pons computed tomography brain showed increased focal uptake of tracer in the left frontal lobe, anteriosuperior to anterior cingulate gyrus [Figure 4]. After 10 days of treatment, the patient improved significantly. Cervical lymph nodes resolved. Eye movements were full. Cerebellar signs and blepharospasm disappeared [Videos 3 and 4]. Gait became normal. The patient was followed up at 1-3 months without any residual neurological deficits. Magnetic resonance imaging brain done after 1 month of treatment showed complete resolution of lesions in midbrain and pons [Figure 5].

\section{Discussion}

Kikuchi-Fujimoto disease usually has a benign course which resolves spontaneously. Kikuchi-Fujimoto disease is described as viral infection with autoimmune process and genetic susceptibility. ${ }^{[4]}$ There are few case reports where the fatal outcome has been described in Kikuchi-Fujimoto disease. ${ }^{[5]}$ The disease affects females ${ }^{\left[{ }^{[6]}\right.}$ usually and mean age is around 25 years. ${ }^{[7]}$ It was reported more frequently in pediatric and adolescent population than in the elderly population. ${ }^{[8]}$

Disease etiology is unclear. Implicated organisms include Yersinia enterocolitica, Rubella, Parainfluenza virus, Epstein-Barr virus, and Paramyxovirus. ${ }^{[7]}$

Common clinical and laboratory features include fever, leukopenia, skin rash, upper respiratory tract symptoms, cervical lymphadenopathy, enlargement of intraparotid, mediastinal, abdominal, and pelvic lymph nodes. ${ }^{[9]}$ Less common features include hepatosplenomegaly, polyarthralgia.

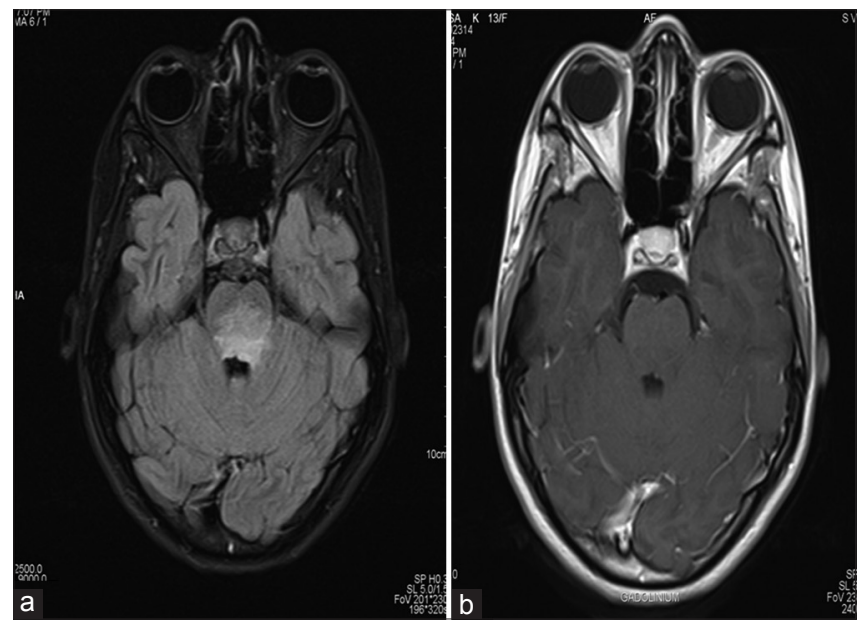

Figure 2: Magnetic resonance imaging of brain (a) fluid attenuated inversion recovery sequences showing hyperintense signal in dorsal pons (b) no postcontrast enhancement in dorsal pons 


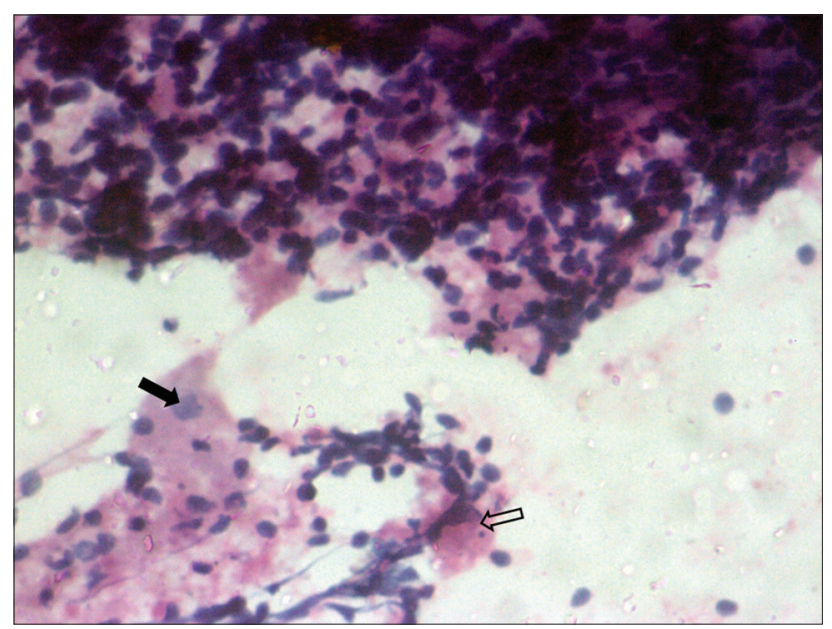

Figure 3: Papanicolaou staining $\times 20$ of cervical lymph node fineneedle aspiration cytology showing lymphocytes, macrophages, foci of necrosis (shaded arrow), and macrophage engulfing karyorrhectic debris (open arrow) which is characteristic of Kikuchi-Fujimoto disease

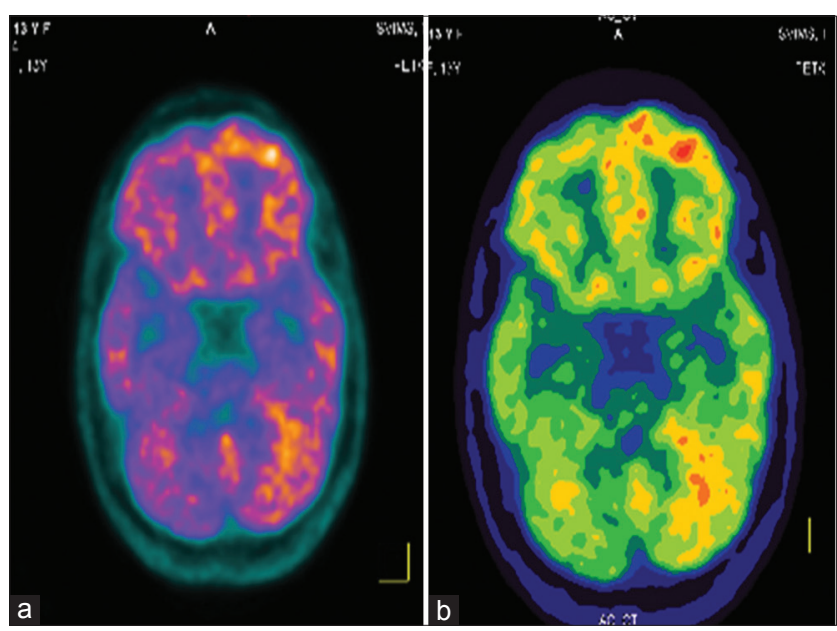

Figure 4: Positron emission tomography/computed tomography of brain showed increased focal uptake of tracer in (a) left frontal lobe, anteriosuperior to anterior cingulate gyrus (b) left frontal and left temporal lobe
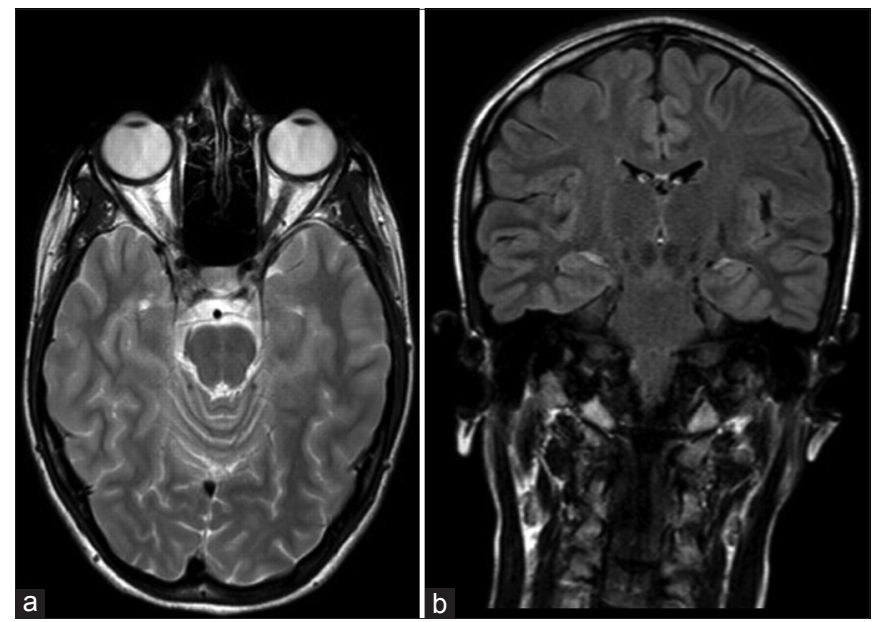

Figure 5: Magnetic resonance imaging of brain (a) T2-weighted sequence, (b) fluid-attenuated inversion recovery sequence showing complete resolution of hyperintensities
Neurological involvement is rare in Kikuchi-Fujimoto disease. Review of the literature showed aseptic meningitis as most reported neurological complication in Kikuchi-Fujimoto disease. ${ }^{[10]}$ Aseptic lymphocytic meningitis was described in $9.8 \%$ cases in Japan. But in India, there are very few reports of neurological complications in Kikuchi-Fujimoto disease. Only five cases of encephalitis have been reported worldwide as a complication in Kikuchi-Fujimoto disease. Encephalitis reported till now was limited to the brainstem, temporal lobes, and cerebellum. ${ }^{[11]}$ Cerebellar ataxia, diplopia, and confusion have also been described in few cases. Unusual and rare features in our case were an association of blepharospasm and brainstem encephalitis with Kikuchi-Fujimoto disease.

Blepharospasm secondary to infectious process is very rare. Regions of the brain responsible for blepharospasm are uncertain. Functional imaging done in patients with blepharospasm pointed to abnormal activity in the brainstem, cerebellum, caudate nucleus, thalamus, somatosensory, and cingulate cortices. ${ }^{[12]}$ In our case, PET scan showed increased focal uptake in anterior cingulate cortex, which is the probable site of origin of blepharospasm. A thorough review of the literature did not show any case reports of brainstem encephalitis and secondary blepharospasm in Kikuchi-Fujimoto disease in India. Our case will be the first reported case of Kikuchi-Fujimoto disease from India with brainstem encephalitis and secondary blepharospasm.

\section{Conclusion}

Kikuchi-Fujimoto disease is often misdiagnosed as tuberculosis or lymphoma if it presents with associated neurological manifestations. Our case reports the possibility of neurological manifestations such as secondary blepharospasm and brainstem encephalitis in Kikuchi-Fujimoto disease. It can be diagnosed by cervical lymph node biopsy, thereby avoiding unnecessary treatment. Our case report highlights consideration of Kikuchi-Fujimoto disease as a differential diagnosis in any case of acute encephalitis syndrome with cervical lymphadenopathy.

\section{Financial support and sponsorship} Nil.

\section{Conflicts of interest}

There are no conflicts of interest. 


\section{References}

1. Kikuchi M. Lymphadenitis showing focal reticulum cell hyperplasia with nuclear debris and phagocytosis. Nippon Ketsueki Gakkai Zasshi 1972;35:379-80.

2. Fujimoto Y, Kosima Y, Yamaguchi K. Cervical subacute necrotizing lymphadenitis. A new clinicopathological agent. Naika 1972;20:920-7.

3. Mohan A, Reddy MK, Phaneendra BV, Chandra A. Aetiology of peripheral lymphadenopathy in adults: Analysis of 1724 cases seen at a tertiary care teaching hospital in southern India. Natl Med J India 2007;20:78-80.

4. George TI, Jones CD, Zehnder JL, Warnke RA, Dorfman RF. Lack of human herpesvirus 8 and Epstein-Barr virus in Kikuchi's histiocytic necrotizing lymphadenitis. Hum Pathol 2003;34:130-5.

5. Chan JK, Wong KC, Ng CS. A fatal case of multicentric Kikuchi's histiocytic necrotizing lymphadenitis. Cancer 1989;63:1856-62.

6. Kuo TT. Kikuchi's disease (histiocytic necrotizing lymphadenitis).
A clinicopathologic study of 79 cases with an analysis of histologic subtypes, immunohistology, and DNA ploidy. Am J Surg Pathol 1995;19:798-809.

7. Kucukardali Y, Solmazgul E, Kunter E, Oncul O, Yildirim S, Kaplan M. Kikuchi-Fujimoto disease: Analysis of 244 cases. Clin Rheumatol 2007;26:50-4.

8. Lin HC, Su CY, Huang SC. Kikuchi's disease in Asian children. Pediatrics 2005;115:e92-6.

9. Bennie MJ, Bowles KM, Rankin SC. Necrotizing cervical lymphadenopathy caused by Kikuchi-Fujimoto disease. Br J Radiol 2003;76:656-8.

10. Sato Y, Kuno H, Oizumi K. Histiocytic necrotizing lymphadenitis (Kikuchi's disease) with aseptic meningitis. J Neurol Sci 1999;163:187-91.

11. Shafqat S, Memon SB, Hyder S, Hasan SH, Smego RA Jr. Brainstem encephalitis with Kikuchi-Fujimoto disease. J Coll Physicians Surg Pak 2003;13:663-4.

12. Neychev VK, Gross RE, Lehéricy S, Hess EJ, Jinnah HA. The functional neuroanatomy of dystonia. Neurobiol Dis 2011;42:185-201. 